\title{
Application of Performance Motion Capture Technology in Film and Television Performance Animation
}

\author{
ZHANG Manyu \\ Dept. Animation of Art, \\ Tianjin Academy of Fine Arts Tianjin , CHINA \\ E-mail: manyu.zhang@vub.ac.be
}

\begin{abstract}
In today's animation films, virtual character animation is a research field developing rapidly in computer graphics, and motion capture is the most significant component, which has brought about revolutionary change for 3D animation production technology. It makes that animation producers are able to drive animation image models directly with the use of actors' performance actions and expressions, which has simplified animation manufacturing operation greatly and enhanced quality for animation production.
\end{abstract}

Keywords- film and television films, motion capture, technology

\section{INTRODUCTION}

With the development of modern computer technology, computer animation technology has developed rapidly. In particular, introduction of 3D concept makes stereoscopic sensation and reality sense much stronger, which conforms to the present world recognized by people more. In today's animation films, virtual human animation is a research field developing rapidly in computer graphics, and motion capture is the most significant component. In addition, it is a kind of high and new technology carrying out real-time capture and digital analysis towards 3D motion trail of the human being, animals, or objects by virtue of motion capture, which can be proved by 3D animation films of European and American countries, such as Avatar, The Adventures of Tintin, Puss in Boots, A Monster in Paris, and Ice Age etc.

\section{MOTION CAPTURE TECHNOLOGY}

Animation production is an item of complicated and time-consuming work. In order to reduce working amount of animation producer in the process of computer 3D animation production, performance animation has been researched and applied further, while motion capture technology is the most important and indispensable in performance animation. Motion capture technology is a kind of technology recording motion of human body and transforming it as digital pattern. In fundamental principles, it records motion and space shift of the human being or animals and drives virtual characters to move with these data, so as to achieve results that are more fluent and accurate than manual animation ${ }^{[1]}$. As for motion capture in early stage, it only records actors' body language, and face expression depends on later drawing of animators. However, film Rise of the Apes has developed dramatically in face capture, and it has used "face muscular tissue simulation technique" for the first time, so that acting skills of actors can be delivered to viewers maximally. What's more, people realize that "motion capture technology" shall be called as "performance capture technology" increasingly. Orangutan Caesar presented in Rise of the Apes through "motion capture" technology is very classical. Various complicated and subtle expressions and body languages of Caesar's not vanished bestiality and initially emerged human nature have been presented naturally and freshly in the film, which has achieved the excellent realm of mixing the false with the genuine. When Caesar stands in front of Doctor Will in equal gesture, its independence and arbitrariness in its expression with dignity of orangutan has undoubtedly let viewers understand implication of the film name "Rise of the Apes" clearly. In a manner of speaking, it is just the technology of "motion capture" let this film succeed, which also proves that advanced science and technology in Hollywood not only create exaggerated stunts in motion occasion, but also combine technology and the story perfectly.

\section{BASIC COMPOSITION OF PERFORMANCE MOTION CAPTURE TECHNOLOGY}

In film and television performance animation system (Fig1), based on computer graphic principles, we record motion conditions of animated objects in the form of graphs with the use of several video capture devices, and then deal with graphic data with the use of computer. Working processes of motion capture technology system are as follows: write character scripts, draw shooting scripts, digitalize role style design, and make animation model according to requirements for scripts. 


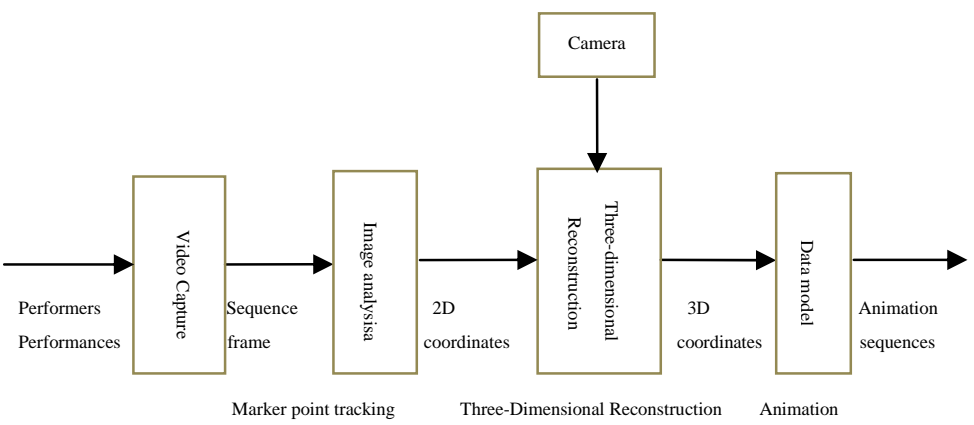

Fig1 animation system

And at the same time, require performers to carry out motion performance, record motion conditions of animated objects in the form of graphs with the use of video capture devices, and then deal with graphic data with the use of computer, so as to generate animation sequence. For the convenience of edition on later stage, performers are required to put on blue dresses and to stick on special signs or luminous points in key positions of their body, such as joints, hips, elbows, and wrists, and vision system can identify and dispose these signs. Conduct real-time video capture with the use of several video cameras, and motion conditions of mark points in each frame can be seen from sequence pictures gained from cameras. Therefore, a continuous motion trail of a specific point with time variation can be acquired, and then motion trails of these points can be restored as motions of skeleton pattern through $3 \mathrm{D}$ reconstruction technology ${ }^{[1][2] \text {. }}$

\section{APPLICATION OF PERFORMANCE MOTION CAPTURE TECHNOLOGY IN FILM AND TELEVISION ANIMATION PRODUCTION}

In the early days, production of 3D animation mainly depends on adjustment of animators frame by frame, with long time consumption, complexity, and low efficiency. Therefore, time consumption for motions produced with the use of 3D animation software was large in the past, and production for some motions is clumsy and rigid ${ }^{\text {[3]. }}$ It is not difficult to see this phenomenon in some films. However, many vivid animation characters are created on the basis of motion capture technology, and it captures motions of actors, athletes, and dancers. Motions of actors are not limited, with fluent and natural motions, which reduce time for animators to adjust motion ${ }^{[4]}$.

As for gorillas in Rise of the Apes shown in the big screen in 2011, none of them is a real gorilla, and they are animated motion-capture performers in the rear of these hairy living beings; machinery fighters shaking fists naturally and fluently in Real Steel are also actors themselves with blue dresses and stilts; in The Adventures of Tintin, it is cartoon pattern with extremely natural motions and expressions, which is also achieved by motion capture of real actors.

The most classical work for the use of motion capture in films is Lord of the Ring, and strange-looking Gollum (Fig2)

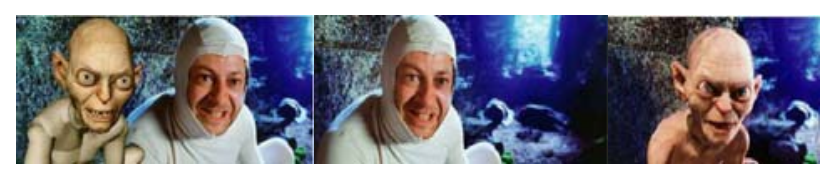

Fig2 how to make Gollum production process

awarded the nomination of "the best actor in a supporting role" of Oscar in the same year. At that time, at the time of shooting Gollum, all scenes with Gollum shall be manufactured for three times: firstly, it is real shooting. Andy Serkis in white performs with actors; secondly, in the computer, Andy Serkis is erased, so as to place Gollum; thirdly, individual performance of Andy Serkis. He performs in motion-capture dresses with recording spots. Animators make motion modeling for Andy Serkis at first, and then they will transform data to the model of Gollum. As for this kind of production, time consuming is long, with huge expenses.

The film The Polar Express (2004) makes motion capture technology become normalized (Fig3). 


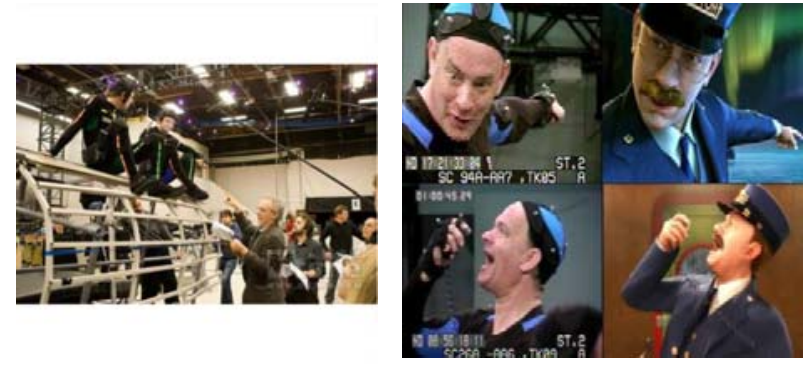

(Fig3) make Polar Express production process

The Polar Express is a complete CG animation film using motion capture from beginning to end for the first time, which also achieves body motion capture and face performance capture jointly for the first time. For this reason, this film has been recorded in Guinness World Records (2006). And at the same time, at the site of shooting The Polar Express, there are more than 60 video cameras, calculating and capturing body performance and face performance of several actors at a time, which achieves the animation character whose motion is most close to real person.

With gradually matured motion capture technology, there is a Creation completed with motion capture technology in every year. Tall King Kong and fierce and terrible dinosaurs in King Kong (2005), Pirates of the Caribbean II (2006), and Beowulf (2007) directed by Robert Zemeckis reveals CG human characters. However, The Curious Case Of Benjamin Button (2008) lets CG character of real person peak, which is the only performance capture approved by Oscar. Up to now, we can still say that CG human character performance in this film is the most excellent. In previous films with motion capture, there are still some issues which can not be broken through. However, in the 3D film named Avatar, common passive optics motion capture system is adopted to capture data for body movement, and video motion capture technology is also be used. In addition, facial expression capture helmets which are worn on the head of actors are researched and developed. Through respective capture towards body data and face data, Avatar effectively overcomes the dilemma that several groups of data are not captured at the same time formerly ${ }^{[5]}$. With several sets of motion capture systems capture at the same time and in real time, brave and resolute Avatar, a mixed-blood creature is created. The meaning of Avatar in the aspect of motion capture is that it has achieved unprecedented and lifelike visual life, which has eliminated the sense of isolation with viewers brought by digital characters in the past and stepped over "Uncanny Valley" successfully. Rise of the Apes shown in 2011 is a high-end film where motion capture walks out of photo studio. In addition, motion capture technology of Rise of the Apes is perfect combination of technology of Avatar and motion capture technology. It can be said that the motion capture technology of Rise of the Apes has basically solved all issues related to motion capture: body real-time capture, onthe-spot demonstration, synchronous capture in the face, and not needing for additional performance time. Furthermore, the team has improved identification system on that basis. Actors' performances can be captured perfectly only through installing about 50 light sources in the face. They make animated gorillas with the use of matching of Solver software and manufacturing of skin line ${ }^{[6]}$. Rise of the Apes not only has the meaning in motion capture technology, but also lets everybody value this technology in perception.

\section{CONCLUSION}

With continuous development in digital film production technology, it is required to enhance motion capture technology continuously. Motion capture technology has realized a visualization way simulating technical motions in 3D way, so that animation producers can further perfect computer vision technology for effectiveness and practicability of system.

\section{REFERENCES}

[1] Qu Yi and Li Cunhua. Research on Application of Motion Capture Technology in Film and Television Animation Production. Information Technology, 2006.

[2] Jin Gang, Li Dehua, and Zhou Xueyong. Motion Capture Technology in Performance Animation. Journal of Image and Graphics, 2000.

[3] Huang Boshi and Chen Fumin, Motion Capture and Its Application on Animation Production. Computer Engineering, 2005.

[4] Qiu Wangbiao and Li Chao, Research on Protected Methods of Dances and Arts of the Chinese Minorities Based on Motion Capture Technology. Journal of Beijing Dance Academy, 2009

[5] Revelation of Avatar Brought to Chinese Animation. Modern Communication (Journal of Communication University of China), 2010

[6] Ron Fischer. Motion capture and motion editing [M]. Cambridge :Cambridge University Press ,2000. 